\title{
Application of the Entropy of Approximation for the nonlinear characterization in patients with Chagas Disease
}

\author{
M. Vizcardo ${ }^{\dagger}$, A. Ravelo ${ }^{\ddagger}$, M. Manrique ${ }^{\dagger}$, P. Gomis $^{\S}$ \\ ${ }^{\dagger}$ Escuela Profesional de Física, Universidad Nacional de San Agustín de Arequipa, Perú \\ ${ }^{\ddagger}$ Instituto for Technological Develoment and Innovation in Communications, Universidad de Las \\ Palmas de Gran Canaria, Spain \\ $\S$ Universitat Politècnica de Catalunya-Barcelona Tech (UPC), Spain
}

\begin{abstract}
Chagas disease American trypanosomiasis is caused by a flagellated parasite: Trypanosoma cruzi, transmitted by an insect of the genus Triatoma and also by blood transfusions. In Latin America, the number of infected people is approximately 6 million, with a population exposed to the risk of infection of 550000. It is our interest to develop a non-invasive and low-cost methodology, capable of detecting any early cardiac alteration that also allows us to see dysautononia or dysfunction within 24 hours and with this it could be used to detect any cardiac alteration caused by T early Cruzi. For this, we analyzed the 24hour Holter ECG records in 107 patients with ECG abnormalities (CH2), 102 patients without ECG alterations (CH1) who had positive serological results for Chagas disease and 83 volunteers without positive serological results for Chagas disease (CONTROL). Approximate entropy was used to quantify the regularity of electrocardiograms (ECG) in the three groups. We analyzed 288 ECG segments per patient. Significant differences were found between the CONTROL-CH1, CONTROL-CH2 and CH1CH2 groups.
\end{abstract}

\section{Introduction}

Chagas disease is an endemic disease, which is caused by a flagellated parasite: Trypanosoma cruzi (T. cruzi), transmitted by an insect of the genus Triatoma, Chagas disease can also be acquired by blood transfusions. According to WHO in Latin America, the number of infected people is approximately 6 million, with a population exposed to the risk of infection of 568,000[1]. Previous studies show that $40 \%$ of the population infected with T. cruzi have cardiac compromise[2, 3,11]. In Chagas disease we can distinguish two phases: an initial acute phase of infection and a prolonged intermediate chronic phase, where the disease is often clinically silent and asymptomatic. It is our interest to develop a low-cost non-invasive methodol- ogy that allows to distinguish dysautononia or dysfunction in the course of 24 hours and with this it could be used as an early marker that shows cardiac alterations produced by T. cruzi

\section{Database}

For this work, we have used the electrocardiogram (ECG) database of the Instituto de Medicina Tropical (IMT) of the Universidad Central de Venezuela. The patients and volunteers underwent the following tests: clinical evaluation, positive Machado-Gerreiro serological test, chest x-rays, echocardiogram, electrocardiogram and Holter recording ( 24 hours). They were classified into three groups: 83 healthy volunteers called the Control group; 102 patients infected with only the positive serological test of Machado-Gerreiro (clinical evaluation, chest Xrays, echocardiogram, electrocardiogram and Holter were normal) called group $\mathrm{CH} 1$ and 107 seropositive patients with incipient heart disease, atrioventricular block (BAV) involvement first grade, sinus bradycardia (BS) or right bundle branch block of His (BRDHH), all of which were not being treated with medications, called the $\mathrm{CH} 2$ group. All were outpatients and informed consent was obtained from all of them.

\section{Method}

\section{1. $\quad$ Preprocessed}

We will use the 24-hour ECG holter register (Rozzin model 151 with two leads), which we will divide into 288 segments (framers) corresponding to 5 minutes of ECG (approximately 300 beats) for each volunteer or patient. ECG signals were recorded at $500 \mathrm{~Hz}$ with 12 bits of resolution, one channel was recorded. Because we will use a method based on the regularity of the data, no filter was used. 


\subsection{Feature extraction}

In statistics, an approximate entropy (ApEn) is a technique used to quantify the amount of regularity and the unpredictability of fluctuations over time-series data[4,5].

The approximate entropy (ApEn) was developed by Steve Pincus [4, 5] based on the entropy of KolmogorovSinai KS, proposed by Grassberger and Procaccia[6] and modified by Takens[7]. Later Richman[8] made a modification of ApEn, which is the sampling entropy (SampEn). Both ApEn and SampEn are based on comparisons of component-to-component embedment vectors $(m=2)$ and with a threshold of $20 \%$ of the standard deviation of the ECG $(r=0.20)$. The difference between the entropies is that ApEn does not take into account the parisons of the embedment vectors with itself and the way to calculate the logarithm[9]. To calculate ApEn:

$$
\operatorname{ApEn}(m, r, N)=-\frac{1}{N-m} \sum_{i=1}^{N-m} \log \left(\frac{A_{i}}{B_{i}}\right)
$$

where $B_{i}$ are the vectors of embedding dimension $m$ and $A_{i}$ are the vectors of dimension of embendding $m+1, r$ is the threshold that is typically $20 \%$ of the value of the standard deviation and $N$ is the number of elements.

SampEn is calculated as:

$$
\operatorname{SampEn}(m, r, N)=-\log \left(\frac{\sum_{i=1}^{N-m} A_{i}}{\sum_{i=1}^{N-m} B_{i}}\right)=-\log \left(\frac{A}{B}\right)
$$

similar a ApEn $m, r$ and $N$. The ApEn and SampEn was applied at 288 framers each frame corresponds to $5 \mathrm{~min}$ utes per patient

\subsection{Kruskal Wallis test and logistic regres- sion}

The Kruskal Wallis test will be used to find significant differences $(p-$ value $<0.05)$ between the groups: Control-CH1, Control-CH2 and $\mathrm{CH} 1-\mathrm{CH} 2$ in the 288 framer respectively. A logistic regression is also used to evaluate the circadian profiles of the average values of ApEn, SampEn.

\section{Results}

In Figure 1 we can see that, on average, the three groups have an increase in the values of ApEn and SampEn of frame 50 that would correspond to 4:00 hours and then a decrease of frame 250 corresponding to 20:00 hours, This result coincides with[9] where tachograms are used.

\subsection{Approximate Entropy (ApEn)}

- The average values of the circadian profiles of the ApEn of group $\mathrm{CH} 1$ (red) always remain below the average values of the control group (blue)

- We observe that the average values of the circadian profiles of ApEn of the control and $\mathrm{CH} 1$ groups show the greatest difference in framer 50 and 175 corresponding to 5:00 and 14:00 hours.

- As for the average values of the circadian ApEn profiles of the $\mathrm{CH} 2$ group (black) they remain below the average values of the control group (blue) after the framer 90

- We also observe that the average values of the circadian ApEn profiles of the control and $\mathrm{CH} 2$ groups show the greatest difference in framer 100 and 175 corresponding to $8: 00$ and $14: 00$ hours.
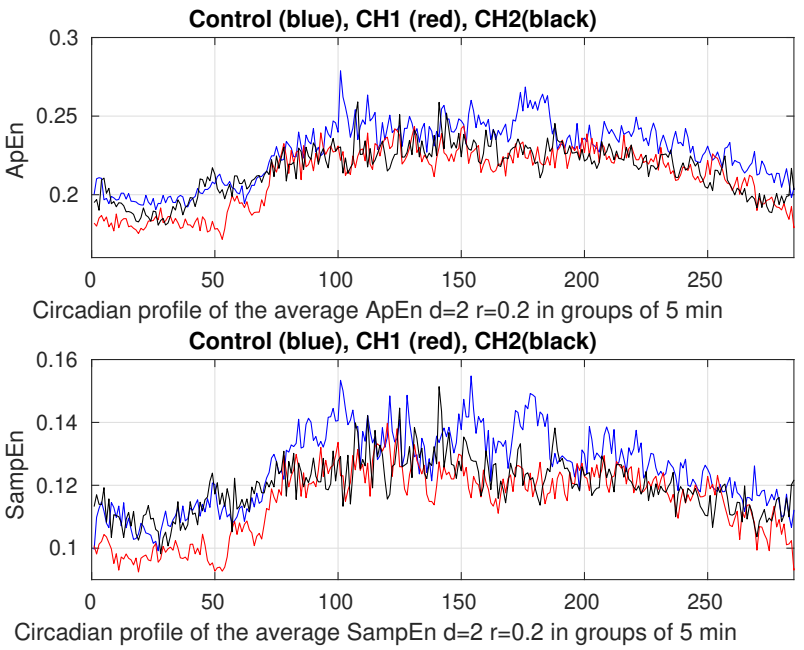

Figure 1. The average values of ApEn and SampEn of 24 hours (288 frames)

As for the Kruskal Wallis test figure 2

- We found significant differences between the Control and $\mathrm{CH} 1$ groups in several frames, but we can highlight in frames 50, 100 and 150-180 corresponding to 4:00, 8:00 12:00-15:00 hours.

- With respect to the control and the $\mathrm{CH} 2$ group, there are significant differences in several frames, we can highlight the 100 and 175 frames corresponding to 8:00 and 14:00 hours.

- For $\mathrm{CH} 1-\mathrm{CH} 2$ there are significant differences in the frame of 50 corresponding to 4:00 hours

We have used logistic regression to discriminate between the Control and $\mathrm{CH} 2$ groups of ApEn for this, $50 \%$ of the frames are chosen at random (144) for training and the other $50 \%$ for validation, finding $80 \%$ specificity and $60 \%$ sensitivity in framers 100 and 175 corresponding to 8:00 and 14:00 hours respectively figura 4 

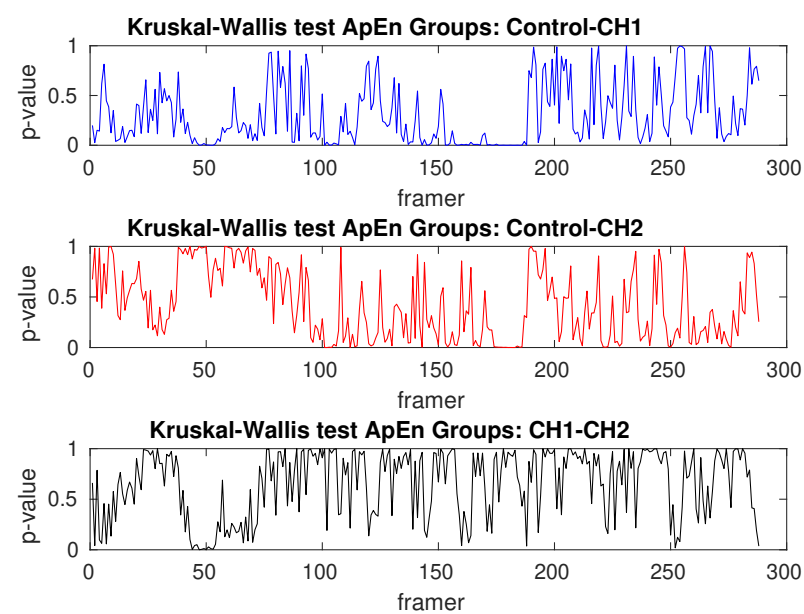

Figure 2. Kruskal-Wallis test of ApEn of the 288 framer

\subsection{Sample Entropy (SampEn)}

- The average values of the circadian profiles of the SampEn of group CH1 (red) always remain below the average values of the control group (blue)

- We observe that the average values of the circadian profiles of SampEn of the control and CH1 groups show the greatest difference in framer 50 and 175 corresponding to 5:00 and 14:00 hours.

- As for the average values of the circadian SampEn profiles of the $\mathrm{CH} 2$ group (black) they remain below the average values of the control group (blue) after the framer 75

- We also observe that the average values of the circadian SampEn profiles of the control and $\mathrm{CH} 2$ groups show the greatest difference in framer 100 and 175 corresponding to 8:00 and 14:00 hours.

As for the Kruskal Wallis test figure 3

- We found significant differences between the Control and $\mathrm{CH} 1$ groups in several frames, but we can highlight in frames 50,100 and $150-180$ corresponding to $4: 00,8: 00$ 12:00-15:00 hours.

- With respect to the control and the $\mathrm{CH} 2$ group, there are significant differences in several frames, we can highlight the 100 and 175 frames corresponding to 8:00 and 14:00 hours.

- For CH1-CH2 there are significant differences in the frame of 50 corresponding to 4:00 hours

We have used logistic regression to discriminate between the Control and CH2 groups of SampEn for this, 50\% of the frames are chosen at random (144) for training and the other $50 \%$ for validation, finding $80 \%$ specificity and $60 \%$ sensitivity in framers 100 and 175 corresponding to 8:00
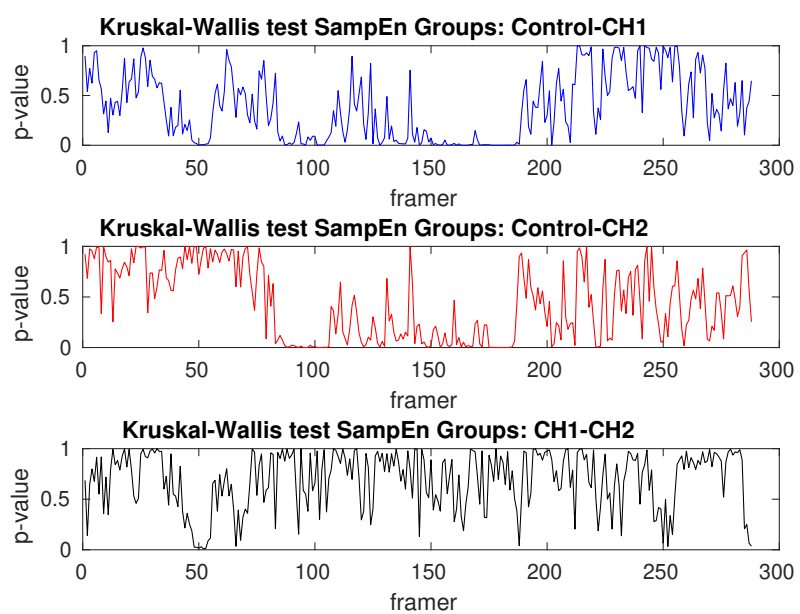

Figure 3. Kruskal-Wallis test of SampEn of the 288 framer
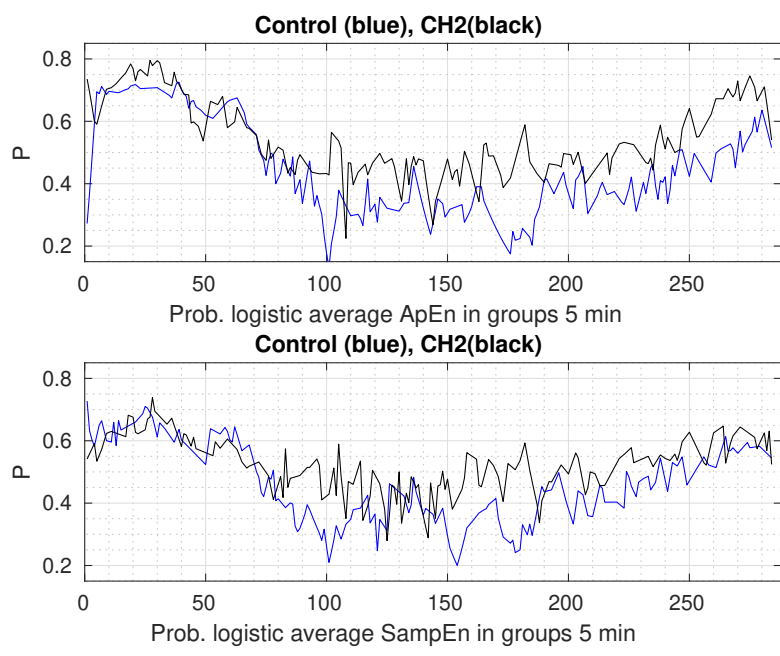

Figure 4. Logistic classifier of the average values of ApEn and SampEn of the 288 framer

and 14:00 hours respectively figura 5

\section{Discussion and conclusions}

The Apen and SampEn of ECG can be used as a measure of heart rate variability similar to HVR, as well as other entropies[10]

The CH1 group, its average values of ApEn and SampEn, show a decrease or enervation in their baroreflector response or autonomic nervous system.

The CH2 group its average values of ApEn and SampEn in some framers show an increase with respect to the Control group this would show a dysautonomy or lack of control of their autonomic nervous system. 

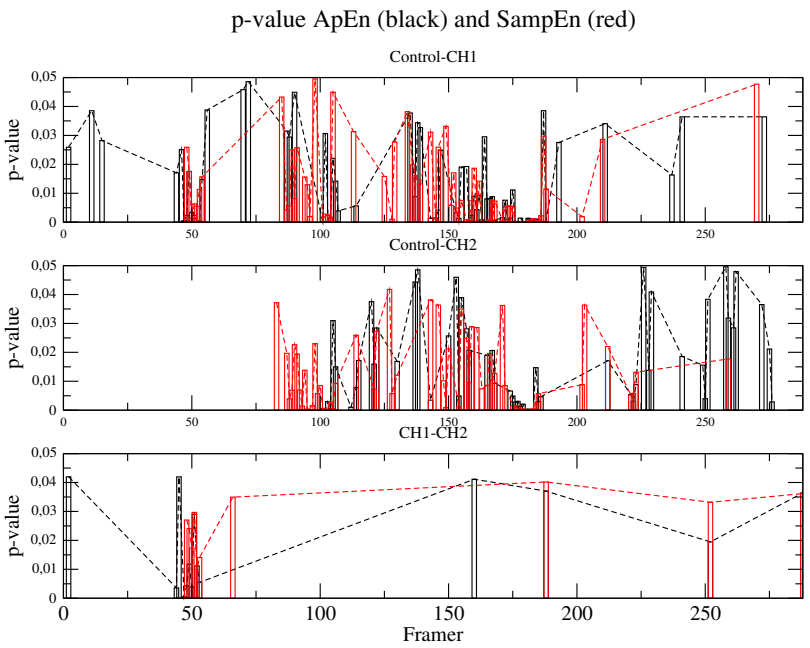

Figure 5. p-value less than 0.05 of ApEn and SampEn

Figure 5 shows the p-values less than 0.05 that would be the framers or moments where the three groups are significantly different, that is to say the Control group and the $\mathrm{CH} 1$ group are significantly different in 04:00, 08:00, 10:00-14:00 hours. The Control group and $\mathrm{CH} 2$ are significantly different from 08:00, 12:00 and 14:00 hours. Finally there is a significant difference between the groups $\mathrm{CH} 1$ and $\mathrm{CH} 2$ only at 04:00 hours.

\section{Acknowledgements}

Universidad Nacional de San Agustin de Arequipa Perú and Instituto for Technological Develoment and Innovation in Communications, Universidad de Las Palmas de Gran Canaria, Spain

\section{Conflicts of Interest}

The authors declare no conflict of interest

\section{References}

[1] World Health Organization, The World Health Report, 2017, 2:75,9-16, http://www.who.int/wer

[2] Moleiro F. and I. Mendoza, Circulation, 1978, 113

[3] Moleiro F, Mendoza I, Miocardiopatia Chagásica Crónica. Estudio Epidemiológico, Acta Cient. Venez. 1980, 31:66

[4] Pincus S, Gladston I, A Regularity Statistic for Medical Data Analisys, Journal of Clinical Monitoring October, 1991. 1980, Vol 7 No 4

[5] Pincus S Approximate entropy as a measure of system complexity, Proc. Nati. Acad. Sci. USA, 1991, Vol. 88, $2297-$ 2301, Mathematics

[6] Grassberger P, Procaccia I. Estimation of the Kolmogorov entropy from a chaotic signal, Phys Rev A 1983, 28:25912593
[7] Takens S, Invariants related to dimension and entropy, In: Atas do 13 Rio de Janeiro, Col. Brasiliero de Matematicas, 1983

[8] Richman J., Moorma J., Physiological time-series analysis using approximate entropy and sample entropy, Am J Physiol Heart Circ Physiol, 278: H2039H2049, 2000.

[9] Vizcardo M., Ravelo A., Computing in Cardiology Conference (CinC), 2018

[10] Vizcardo M, et.al., Nonlinear characterization of ECGs in patients with Chagas' disease, 2019, Biomedical Physics \& Engineering Express, doi $=10.1088 / 2057-1976 /$ ab03f7, IOP Publishing, vol 5, number 2, pag 025042.

[11] Hagar J. M. and Rahimtoola S. H., N. Eng. J. Med., 325, 763, 1991.

[12] Guzzetti S., et.al., Impaired heart rate variability in patients with chronic Chagas' disease., Am. Heart J. 1991, 121:1727

[13] Loyo J, et.al., DV:Effect of postural changes in the autonomic balances in latent or indeterminate phase of Chagas Disease, Chest 1995, 3:108

[14] Liebovitch L. S., et.al., Phys. Rev. E, 59, 3, 1999.

[15] Vizcardo M., et.al, Analysis of the heart rate variability and stratification of the risk of cardiac patients with Chagas disease, Computers in Cardiology, 2007.

[16] Wales D. J., Calculating the rate of loss of information from chaotic time series by forecasting, Nature 350, 485, 1991.

[17] Ravelo-García A. G., et.al. Symbolic dynamics marker of heart rate variability combined with clinical variables enhance obstructive sleep apnea screening, Chaos 24, 024404, 2014.

[18] Ravelo-García A. G., et.al., Application of the Permutation Entropy over the Heart Rate Variability for the Improvement of Electrocardiogram-based Sleep Breathing Pause Detection, , Entropy, 2015, 17, 914-927; doi:10.3390/e17030914

[19] Merejo Pea C. M. and et.al., Dysautonomy in different death risk groups (Rassi score) in patients with Chagas heart disease, Pacing Clin Electrophysiol, 2018, 41:238245.

[20] Caeiro A. , Iosa T., Chronic Chagas' disease: possible mechanism of sinus bradycardia. Can J Cardiol, 1994, 10:765-8

[21] Octavio J, et.al., Perfiles circadianos de la frecuencia cardíaca y de su variabilidad instantánea en una población de pacientes con infección chagásica crónica. Rev Esp Cardiol., 2004, 57(2):130-7

[22] Ravelo-García, et.al, Cepstrum feature selection for the classification of sleep apnea-hypopnea syndrome based on heart rate variability. In Computing in Cardiology Conference (CinC), 2013 (pp. 959-962). IEEE.

[23] Ravelo-García, et.al, An approach to the enhancement of sleep apnea detection by means of detrended fluctuation analysis of RR intervals. In Computing in Cardiology Conference (CinC), 2014 (pp. 905-908). IEEE.

Address for correspondence:

Miguel Vizcardo Cornejo

Calle Santa Catalina N 117 Arequipa, Peru

mvizcardoc@unsa.edu.pe 